\title{
A COMPARISON OF ALTERNATIVE ASSAYS TO \\ MEASURE DNA DAMAGE IN STALLION SPERMATOZOA: TUNEL TEST VERSUS 'NICOLETTI ASSAY'
}

\author{
Balázs KAKASI $^{1 *}$, Szabolcs NAGY ${ }^{2}$, László PÁL ${ }^{2}$, Gyula E. CzIMBER $^{3}$ \\ and Ferenc HuSVÉTH ${ }^{2}$ \\ ${ }^{1}$ Department of Limnology, Institute of Environmental Sciences, University of Pannonia, \\ Wartha Vince u. 1, H-8200 Veszprém, Hungary; ${ }^{2}$ Department of Animal Sciences and \\ Animal Husbandry, Georgikon Faculty, University of Pannonia, Keszthely, Hungary; \\ ${ }^{3}$ BioCzinov Ltd., Mosonmagyaróvár, Hungary
}

(Received 22 August 2014; accepted 29 October 2014)

The aberrations of sperm DNA may cause various problems and have negative consequences on fertility. These influence embryonic development or might lead to early embryo loss. Sperm Chromatin Structure Assay (SCSA) is the flow cytometric method most often used for the detection of DNA lesions; however, some studies using that method reached confusing conclusions. The aim of this pilot study was to adjust and compare two alternative tests, namely the TUNEL test and the Nicoletti assay. The above-mentioned two flow cytometric methods capable of detecting the fragmented DNA of sperm were tested on 12 frozen-thawed stallion semen samples. The TUNEL test demonstrated much higher DNA fragmentation ratio than the Nicoletti assay (mean \pm SD: $30.77 \pm$ $13.03 \%$ vs. $1.93 \pm 0.89 \%$, respectively). A fluorescent microscopic check of the samples showed that TUNEL labelled the plasma membrane and the mitochondria in a nonspecific way, rather than detecting only the fragmented DNA, thus eventually resulting in a false positive sign. The Nicoletti assay is simpler, quicker and does not detect nonspecific binding; however, further analyses are required to determine its diagnostic value.

Key words: Flow cytometry, TUNEL test, Nicoletti assay, stallion semen, DNA fragmentation

The success of fertilisation depends on many properties including the integrity of sperm DNA (Acharyya et al., 2005). Spermatozoa with fragmented DNA can be fully functional and are able to fertilise but have an increased chance to impair the fertilisation process or embryo development (Ahmadi and $\mathrm{Ng}$, 1999; Evenson, 1999; Fatehi et al., 2006).

*Corresponding author; E-mail: balazs.kakasi@gmail.com; Phone: 0036 (83) 545-349; Fax: 0036 (83) 545-107 
One of the flow cytometric methods most often used for the measurement of DNA fragmentation is the Sperm Chromatin Structure Assay (SCSA; Evenson et al., 1980). The test uses the acridine orange fluorescent dye to mark the DNA content of the cells, and measures the metachromatic shift from green to red fluorescence, which gives information about the chromatin structure, including DNA fragmentation (Evenson, 2013). In a recent study, Nagy et al. (2013) have shown that SCSA could not accurately differentiate between subfertile and fertile individuals of the tested bull population. In addition, there are flow cytometer companies which do not recommend using this dye in their devices (or suggest special care when using it), because stain residues may remain in the tubing of the instrument (Beckman Coulter technical support, personal communication). Moreover, the biological background of the assay was questioned (van der Schans et al., 2000).

The terminal-deoxynucleotidyl-transferase-mediated dUTP nick end-labelling (TUNEL) test is based on antibody reactions. The TdT (terminal deoxynucleotidyl transferase) binds to the 3'-hydroxyl ends of the DNA. Normally there are two 3'-hydroxyl ends in an intact DNA, but every strand break increases the number of possible TdT-binding nicks. After the TdT binds to the free nick ends, BrdUTP (5-brome-2'-deoxyuridine-5'-triphosphate) is added, which marks the end of the fragments. In the final step a fluorescent dye conjugated anti-BrdUTP antibody is added, and thus the DNA fragmentation of the cells can be accurately measured with a flow cytometer or a microscope. The test is one of the most popular methods for detecting apoptotic cells and DNA fragmentation. There are several reports on the use of this test on various cells and tissues (Oberhaus, 2003). Although the test is popular because of its microscopic and even flow cytometric applicability and simplicity, some authors have raised concerns about the limitations of its sensitivity and selectivity (Evenson and Wixon, 2006).

Nicoletti et al. (1991) described a simple method suitable for use in flow cytometric analyses. The test uses the fluorogenic dye propidium iodide (PI) to measure DNA fragmentation and apoptosis in cells. This dye binds stoichiometrically to nucleic acids in the DNA, so the measured fluorescence intensities of the cells can be used for determining the DNA content and the degree of DNA fragmentation. The intact DNA remains in the cells, resulting in a defined peak on the intensity histogram, but the small, fragmented DNA can leak out of the cells, and cells with DNA loss show a subdiploid (or in the case of spermatozoa a subhaploid) fluorescent signal. The assay has been applied successfully on several cell types (Riccardi and Nicoletti, 2006; Winkle et al., 2009). 


\section{Materials and methods}

Samples

Frozen sperm samples of 12 randomly selected stallions were stored in $500-\mu 1$ straws in liquid nitrogen. The frozen semen samples were thawed in a $37^{\circ} \mathrm{C}$ water bath for $60 \mathrm{sec}$, and then transferred to 2-ml Eppendorf tubes. Different straws of the same samples were thawed for the two tests.

\section{TUNEL assay}

The flow cytometric analysis of TUNEL test was carried out with the commercially available APO-BrdUTM TUNEL Assay Kit with Alexa Fluor ${ }^{\circledR} 488$ anti-BrdU (A23210, Invitrogen, Carlsbad, CA, USA). In this study the attached assay protocol was followed, with slight modifications. Briefly, $1-2 \times 10^{6}$ cells were suspended in $0.5 \mathrm{ml}$ PBS (Phosphate Buffered Saline, P-4417, SigmaAldrich, St Louis, MO, USA), then $1.5 \mathrm{ml}$ 1\% paraformaldehyde was added and incubated for $15 \mathrm{~min}$ in a $-20^{\circ} \mathrm{C}$ freezer for fixation. The samples were washed twice in $2 \mathrm{ml}$ PBS $(400 \times \mathrm{g}, 10 \mathrm{~min})$, then cells were resuspended in $0.5 \mathrm{ml} \mathrm{PBS}$, permeabilised in $1.5 \mathrm{ml} 70 \%$ ethanol and stored at $-20{ }^{\circ} \mathrm{C}$ overnight. On the next morning the samples were washed three times in the Wash Buffer provided in the TUNEL Kit $(400 \times g, 10 \mathrm{~min})$, then resuspended in $50 \mu \mathrm{l}$ of freshly prepared DNA Labelling Solution (provided in the Kit) and incubated at $37^{\circ} \mathrm{C}$ for $1 \mathrm{~h}$, during which period the samples were shaken at 15-min intervals. When the incubation time expired, $1 \mathrm{ml}$ Rinse Buffer (also provided) was added and the suspensions were washed again (twice, $400 \times g, 10 \mathrm{~min}$ ). Subsequently the cells were resuspended in $100 \mu \mathrm{l}$ freshly prepared Antibody Solution (provided in the Kit) and incubated for $30 \mathrm{~min}$ at room temperature in the dark. Finally $0.5 \mathrm{ml}$ of the propidium iodide/RNase A staining buffer (provided in the Kit) was added and the cells were incubated in dark for an additional 30 min before the measurements were made on a Beckman Coulter FC 500 flow cytometer (Beckman Coulter, Inc., Brea, CA, USA) equipped with a $488 \mathrm{~nm} 20 \mathrm{~mW}$ Ar ion laser.

Fluorescence intensity of the Alexa Fluor 488 conjugate was collected with the FL1 (525 nm BP) detector in logarithmic mode. The sperm population and debris were gated, based on forward scatter versus FL3 (620 nm SP) plot properties. The acquisition record settings were $300 \mathrm{sec}$ or 20,000 events. The files were stored as list mode (LMD) files. List mode files were analysed with Flowing Software (Version 2.5.1, http://www.flowingsoftware.com).

DNA fragmentation was indicated as an increase in the green fluorescence intensity of the Alexa Fluor 488-conjugate. After gating out the debris events and sperm aggregates, one-dimensional FL 1 histograms were drawn. 
Nicoletti assay

The Nicoletti assay quick method was performed as described by Riccardi and Nicoletti (2006). From freshly thawed samples $1-2 \times 10^{6}$ cells were transferred into $1 \mathrm{ml}$ PBS in Eppendorf tubes, and then centrifuged $(400 \times g, 10 \mathrm{~min})$. After washing, the cells were resuspended in $1 \mathrm{ml}$ of a previously prepared Fluorochrome Solution containing $0.1 \%$ sodium citrate (wt/v; cat. no. 27833.237, VWR International, Radnor, PA, USA), 0.1\% Triton X-100 (v/v; cat. no. 900293-1, G-Biosciences, St. Louis, MO, USA), $50 \mathrm{mg} \mathrm{l}^{-1}$ PI (Component B of LIVE/ DEAD Sperm Viability Kit, L-7011, Invitrogen, Carlsbad, CA, USA) in distilled water and incubated at room temperature $\left(20^{\circ} \mathrm{C}\right)$ for $1 \mathrm{~h}$, then measured with the previously described cytometer.

The propidium iodide fluorescence intensity was collected at the FL3 (620 nm SP) detector on logarithmic scale. The files were stored as list mode (LMD) files.

The sperm population was distinguished from all other events based on light scatter properties (FSC vs. SSC), then one-dimensional FL 3 histograms were created. DNA fragmentation was indicated as decreased intensity below the main propidium iodide intensity peak.

\section{Microscopy}

Following the flow cytometric measurements, the samples were checked with an Olympus CX31 fluorescent microscope (Olympus Europe, Hamburg, Germany) equipped with a mercury arc lamp (U-RFLT50) and a fluorescent filter cube turret containing standard FITC and TRITC filters.

\section{Confocal laser scanning microscopy}

TUNEL-labelled spermatozoa (prepared as described above) were analysed by confocal microscopy. One drop of a labelled sperm suspension was put on a Superfrost slide, coverslipped and subsequently analysed on a Bio-Rad MRC 1024 confocal laser scanning microscope (Bio-Rad Laboratories, Inc., Hercules, CA, USA).

\section{Statistics}

The agreement of the two assays was assessed with Bland-Altman method agreement analysis (Bland and Altman, 1986) using Microsoft Excel 2013.

\section{Results}

The average ratio of DNA fragmentation was $30.77 \pm 13.03 \%$ (mean \pm SD) with the TUNEL assay, while the Nicoletti assay showed a DNA fragmentation ratio as low as $1.93 \pm 0.89 \%$. 
The agreement between the two assays was calculated with the BlandAltman method. A Bland-Altman plot was drawn, where the mean differences of the sample sets are represented. The mean difference of the measurements was $28.84 \%$, indicating a large disagreement between the two assays (Fig. 1).

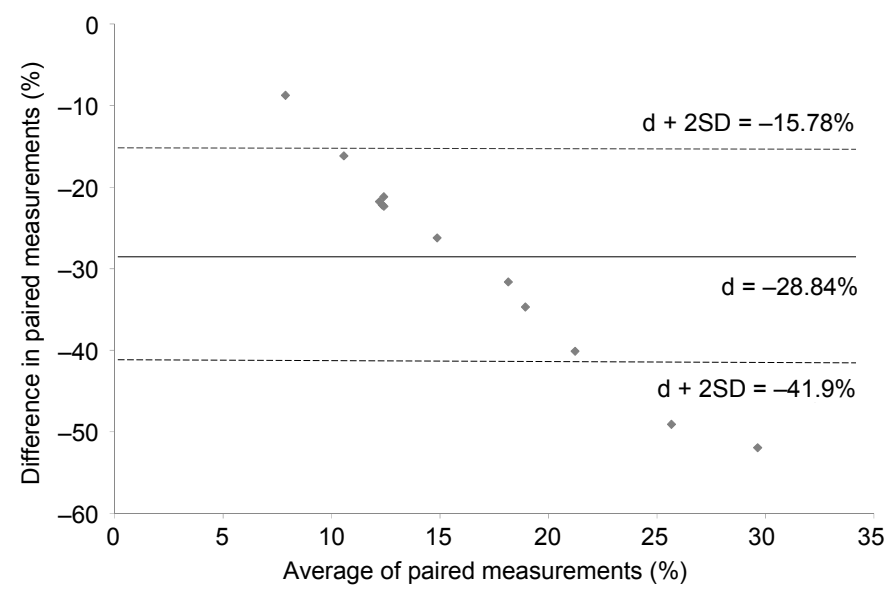

Fig. 1. Method-agreement analysis of cryopreserved stallion sperm DNA damage. The difference between the percentages of sperm DNA fragmentation as assessed by the TUNEL and the Nicoletti method are plotted against their average. The mean of the differences (d) and the limits of agreement $( \pm 2 \mathrm{SD})$ are presented

\section{Discussion}

The aim of the study was to set up and compare the flow cytometric TUNEL and the Nicoletti assays using stallion semen samples. As the results indicated, there were large differences between the two methods in the ratio of DNA damage. The Bland-Altman test showed that the two methods did not agree. This means that, although they both measure DNA fragmentation, they do not provide the same biological information.

The TUNEL test marks the 3'-OH ends of DNA strand breaks (single and double) with antibodies and fluorescent probes. However, the presence of strand breaks does not necessarily mean definite DNA fragmentation. Single and double strand breaks cannot be distinguished from each other with the TUNEL test (Ribeiro et al., 2006). The appearance of DNA single strand breaks is a preceding marker of the fragmentation process, because single strand breaks appear prior to the DNA fragmentation phase (Walker et al., 1997; Iglesias-Guimarais et al., 2013). While the TUNEL test is able to identify an earlier stage of the DNA degrading process, the Nicoletti assay is used for detecting a later appearing apoptotic fragmentation. The propidium iodide dye used in the Nicoletti assay binds tightly to DNA. If the DNA is fragmented, the fragments loaded with fluo- 
rescent dye loaded fragments may flow out of the cells, which finally results in lower-intensity peaks below the intact DNA peak (Riccardi and Nicoletti, 2006).

The large differences obtained between the results of the two methods suggested the possibility of nonspecific labelling with either the TUNEL or the Nicoletti assay. The fluorescent microscopic check of the TUNEL samples showed a nonspecific binding of the Alexa Fluor 488 conjugate to the mitochondria and the plasma membrane, generating a disfigured false positive TUNEL result. Some other authors have also found false positive results with the TUNEL test on various cell types including liver and kidney cells (Stahelin et al., 1998; Pulkkanen et al., 2000). The sensitivity and the selectivity of the assay have also been criticised; moreover, many variants exist and there are no conventional rules on how to carry out the test and evaluate the results (Perreault et al., 2000). The false positive results of TUNEL indicate that conventional flow cytometers are not able to locate the origin of the signal, so fluorescent microscopic control of the results should be a necessary step in every experiment to avoid the interpretation of false data. On the other hand, the Nicoletti assay is much simpler and quicker, and we did not notice any nonspecific binding with its use. However, further analyses are required to determine the diagnostic value of this test for fertility studies in the field.

\section{Acknowledgements}

This study was supported by the project TÁMOP-4.2.2.A-11/1/KONV-2012-0064. The project is supported by the European Union, with co-funding from the European Social Fund.

\section{References}

Acharyya, S., Kanjilal, S. and Bhattacharyya, A. K. (2005): Does human sperm nuclear DNA integrity affect embryo quality? Indian J. Exp. Biol. 43, 1016-1022.

Ahmadi, A. and Ng, S. C. (1999): Fertilizing ability of DNA-damaged spermatozoa. J. Exp. Zool. 284, 696-704.

Bland, J. M. and Altman, D. G. (1986): Statistical methods for assessing agreement between two methods of clinical measurement. Lancet 1,307-310.

Evenson, D. P. (1999): Loss of livestock breeding efficiency due to uncompensable sperm nuclear defects. Reprod. Fertil. Dev. 11, 1-15.

Evenson, D. P. (2013): Sperm chromatin structure assay (SCSA ${ }^{\circledR}$ ). Methods Mol. Biol. 927, 147-164.

Evenson, D. P., Darzynkiewicz, Z. and Melamed, M. R. (1980): Relation of mammalian sperm chromatin heterogeneity to fertility. Science 210, 1131-1133.

Evenson, D. P. and Wixon, R. (2006): Clinical aspects of sperm DNA fragmentation detection and male infertility. Theriogenology 65, 979-991.

Fatehi, A. N., Bevers, M. M., Schoevers, E., Roelen, B. A. J., Colenbrander, B. and Gadella, B. M. (2006): DNA damage in bovine sperm does not block fertilization and early embryonic development but induces apoptosis after the first cleavages. J. Androl. 27, 176-188.

Iglesias-Guimarais, V., Gil-Guinon, E., Sanchez-Osuna, M., Casanelles, E., Garcia-Belinchon, M., Comella, J. X. and Yuste, V. J. (2013): Chromatin collapse during caspase-dependent apop- 
totic cell death requires DNA fragmentation factor, 40-kDa subunit-/caspase-activated deoxyribonuclease-mediated 3'-OH single-strand DNA breaks. J. Biol. Chem. 288, 9200-9215.

Nagy, S., Johannisson, A., Wahlsten, T., Ijas, R., Andersson, M. and Rodriguez-Martinez, H., (2013): Sperm chromatin structure and sperm morphology: their association with fertility in AI-dairy Ayrshire sires. Theriogenology 79, 1153-1161.

Nicoletti, I., Migliorati, G., Pagliacci, M. C., Grignani, F. and Riccardi, C. (1991): A rapid and simple method for measuring thymocyte apoptosis by propidium iodide staining and flow cytometry. J. Immunol. Methods 139, 271-279.

Oberhaus, S. M. (2003): TUNEL and immunofluorescence double-labeling assay for apoptotic cells with specific antigen(s). Methods Mol. Biol. 218, 85-96.

Perreault, S. D., Rubes, J., Robbins, W. A., Evenson, D. P. and Selevan, S. G. (2000): Evaluation of aneuploidy and DNA damage in human spermatozoa: applications in field studies. Andrologia 32, 247-254.

Pulkkanen, K. J., Laukkanen, M. O., Naarala, J. and Yla-Herttuala, S. (2000): False-positive apoptosis signal in mouse kidney and liver detected with TUNEL assay. Apoptosis 5, 329-333.

Ribeiro, G. F., Corte-Real, M. and Johansson, B. (2006): Characterization of DNA damage in yeast apoptosis induced by hydrogen peroxide, acetic acid, and hyperosmotic shock. Mol. Biol. Cell 17, 4584-4591.

Riccardi, C. and Nicoletti, I. (2006): Analysis of apoptosis by propidium iodide staining and flow cytometry. Nat. Protoc. 1, 1458-1461.

Stahelin, B. J., Marti, U., Solioz, M., Zimmermann, H. and Reichen, J. (1998): False positive staining in the TUNEL assay to detect apoptosis in liver and intestine is caused by endogenous nucleases and inhibited by diethyl pyrocarbonate. Mol. Pathol. 51, 204-208.

van der Schans, G. P., Haring, R., van Dijk-Knijnenburg, H. C., Bruijnzeel, P. L. and den Daas, N. H. (2000): An immunochemical assay to detect DNA damage in bovine sperm. J. Androl. 21, 250-257.

Walker, P. R., LeBlanc, J. and Sikorska, M. (1997): Evidence that DNA fragmentation in apoptosis is initiated and propagated by single-strand breaks. Cell Death Differ. 4, 506-515.

Winkle, T., Rosenbusch, B., Gagsteiger, F., Paiss, T. and Zoller, N. (2009): The correlation between male age, sperm quality and sperm DNA fragmentation in 320 men attending a fertility center. J. Assist. Reprod. Genet. 26, 41-46. 